\title{
ANÁLISIS DEL RUIDO BLANCO DE FONDO. INFLUENCIA EN LA ADQUISICIÓN DE UNA SEGUNDA LENGUA
}

\author{
Rosa D'Astoli Santaiti \\ Universidad Internacional Iberoamericana - UNINI (Puerto Rico)
}

\begin{abstract}
Resumen. Este estudio causal-comparativo fue diseñado con el propósito de determinar si el uso de ruido blanco de fondo facilita la adquisición de una segunda lengua en las áreas de comprensión visualauditiva, auditiva y lectora. Este estudio se desarrolló utilizando dos clases de 30 estudiantes cada una. Todos los estudiantes fueron elegidos en forma aleatoria. Una clase fue designada como grupo de control y la otra clase como grupo de experimental. Se definió como variable independiente a la condición de fondo (con sonido/sin sonido) y como variables dependientes los resultados de las evaluaciones de comprensión audio-visual, comprensión auditiva y comprensión lectora. Se estableció como covariable al examen de vocabulario dado al principio de la investigación. Utilizando el programa estadístico SPSS se detectaron 8 valores atípicos; los 52 casos restantes fueron usados para analizar la pregunta de investigación. Utilizando el análisis multivariado de covarianza (MANCOVA) se encontró que en el área de comprensión auditiva, el grupo experimental (con ruido blanco de fondo) tuvo una puntuación significativamente más alta que el grupo control (sin ruido blanco de fondo). En las áreas visual-auditiva y lectora la diferencia fue insignificante. Estos resultados demuestran que el ruido blanco de fondo beneficia el aprendizaje de una segunda lengua en el área de comprensión auditiva. Estos resultados también indican que el ruido blanco de fondo podría utilizarse, cuando sea necesario, como una posible herramienta educativa que no sólo beneficia al alumno; sino también, indirectamente al profesor.
\end{abstract}

Palabras clave: Ruido blanco, resonancia estocástica, TPRS, comprensión visual-auditiva, comprensión auditiva, comprensión lectora.

\section{ANALYSIS OF BACKGROUND WHITE NOISE; ITS INFLUENCE IN THE ACQUISITION OF A SECOND LANGUAGE}

\begin{abstract}
This causal-comparative study was designed to determine if the use of background white noise facilitates second language acquisition, as measured by listening, audio-visual, and reading comprehension. This study was conducted using two classes of 30 students each. All students selected randomly. One class was the control group (no sound) and the second class was the treatment group (sound). The independent variable was the two levels of the background condition (sound/no sound). The dependent variables were the outcome measures of listening comprehension, audio-visual comprehension, and reading comprehension. The covariate was the word recognition test administered at the beginning of the experiment. The data was entered into the Statistical Package for Social Study (SPSS); analysis of data found 8 outliers. After removal of these outliers, 52 cases were used to analyze the research question. A single factor multivariate analysis of covariance (MANCOVA) determined that the treatment group (background sound) scored significantly higher than did the control group (no background sound) on listening comprehension. In the areas of audio-visual and reading comprehension the difference was not significant. These results demonstrate that background white noise benefit listening comprehension in second language acquisition. These results also indicate that white noise background could be used, when necessary, as a possible educational tool that, not only benefits the students; but also, indirectly the teacher.
\end{abstract}

Keywords: White noise, stochastic resonance, TPRS, audio-visual comprehension, listening comprehension and reading comprehension. 


\title{
ANÁLISE DO RUÍDO DE FUNDO BRANCO. INFLUÊNCIA NA AQUISIÇÃO DE UMA SEGUNDA LÍNGUA
}

\begin{abstract}
Resumo: Este estudo causal-comparativo foi projetado para determinar, se o uso de ruído de fundo branco facilita a aquisição de uma segunda língua na compreensão visual-auditiva, audição e leitura. Este estudo foi desenvolvido usando duas aulas de 30 alunos por aula. Todos os alunos foram selecionados aleatoriamente. Uma aula foi designada como grupo controle e a outra aula como grupo de tratamento. Os resultados das avaliações de compreensão audio-visual, compreensão auditiva e compreensão de leitura foram definidos como a variável independente para condição de fundo (com som/sem som) e como variáveis dependentes. O teste de vocabulário feito no início da pesquisa foi estabelecido como covariável. Utilizando o programa estatístico SPSS, foram detectados 8 valores atípicos; os 52 casos restantes foram utilizados para analisar a questão da pesquisa. Utilizando a análise multivariada da covariância (MANCOVA), descobriu-se que, na área de compreensão auditiva, o grupo de tratamento (com ruído de fundo branco) teve um escore significativamente maior do que o grupo controle (sem ruído de fundo branco). Nas áreas de audição-visual e auditiva, a diferença foi insignificante. Esses resultados demonstram que o ruído de fundo branco beneficiam a aprendizagem de uma segunda língua na área do aparelho auditivo. Estes resultados também indicam que o ruído branco pode ser usado, quando necessário, como uma ferramenta educativa que não só beneficia o aluno; mas também, indiretamente facilita ao professor no processo educativo na sala de aula.
\end{abstract}

Palavras chave: ruído branco, ressonância estocástica, TPRS, compreensão audio-visual, compreensão auditiva, compreensão de leitura.

\section{Introducción}

Aun hoy, se enseña de una manera tradicional tratando, en la medida posible, de mantener el silencio. A pesar de que el silencio es considerado como un factor importante para el aprendizaje, la realidad cotidiana demuestra que los estudiantes aprenden de una manera diferente. La música, el ruido, el trabajo de grupo en constante murmullo, no parece afectarles a muchos de los jóvenes de hoy. Posiblemente, el uso de la tecnología ha hecho que los alumnos de hoy difieran con aquellos de años anteriores, lo que conlleva al enseñante a observar y tomar decisiones múltiples. Los alumnos están constantemente rodeados de diferentes estímulos, visuales y/o auditivos, los que posiblemente condicionan el aprendizaje. "Perhaps the most important debate is whether the brain has evolved to utilize random noise in vivo, as part of the "neural code"1 (McDonnell y Abbott, 2009, p.1). A pesar de que, todavía no se sabe si la mente ha evolucionado ante la presencia de los constantes estímulos auditivos, el docente puede observar indiscutiblemente que muchos estudiantes no requieren del silencio para aprender; por el contrario, algunos estudiantes necesitan la presencia de sonidos para concentrarse $\mathrm{y}$ hacer en forma más eficiente su tarea en la clase. Recientes investigaciones han comenzado a vislumbrar que el ruido blanco de fondo podría jugar un papel muy importante en la concentración y aprendizaje de niños con Trastornos de Déficit de Atención (Helps, Bamford, Sonuga-Barke y Söderlund, 2014; Söderlund, Sikström, Loftesnes y Sonuga-Barke, 2010) y niños con Trastornos de Déficit de Atención con Hiperactividad (ADHD, anagrama en inglés) (Sikström y Söderlund,

${ }^{1}$ Tal vez el debate más importante es si el cerebro se ha desarrollado para utilizar in vivo ruido aleatorio, como parte del código neural. 
2007; Baijot, Slama, Söderlund, Dan, Deltenre, Colin y Deconinck, 2016). Generalmente, los estudiantes que sufren de estos trastornos de déficit de atención se encuentran en su mayoría en las clases de estudio de una segunda lengua de niveles básicos. Esta situación que se observa tanto a nivel disciplinario como a nivel académico influye en forma significativa a que estudiantes no continúen con el estudio avanzado de una segunda lengua.

Partiendo de la base que, el ruido blanco, a través del fenómeno de resonancia estocástica, podría ayudar a que un sistema funcione mejor; y considerando que, "optimal levels of white noise will vary from one individual to another"2 (Söderlund et al., 2010, p. 55), se podría declarar que no existe un nivel único que se pueda usar para todas los estudiantes; dado que, cada estudiante posee su propio nivel de ruido interno. Apoyándose en esta idea, el objetivo de esta investigación es averiguar para todos los estudiantes, si el ruido blanco de fondo beneficia el aprendizaje de una segunda lengua; medido en las áreas de comprensión visual-auditiva, auditiva y lectora.

El fenómeno de Resonancia Estocástica es cuando el ruido blanco o aleatorio tiene un efecto positivo. Este fenómeno ha sido observado en sistemas tanto físicos como biológicos (Aledo, 2007; Alvarez Miño y Fajardo Fajardo, 2002; Dolores, 2015; Faisal, Selen Luc y Wolpert Daniel, 2008; Hong, Martin y Saidel, 2006; Lugo, Doti y Faubert, 2008; Mendez-Balbuena, Manjarrez, Schulte-Monting, Huethe, Tapia, HeppReymond y Kristeva, 2012; Mc Donnell y Abbott, 2009; Russell, Wilkens y Moss, 1999; Seo, Kosmopoulos, Enders y Hur, 2014; Toral, 2002). La manera exacta de cómo el ruido blanco o aleatorio beneficia la mente humana no se comprende todavía. Se cree que "a sufficient level of noise may be necessary for normal function in the nervous system, through the process of stochastic resonance"3 (Söderlund et al., 2010, p. 56), afectando la comunicación neuronal (Helps et al., 2014); por ejemplo, "when auditory noise improves visual signal detection" (Söderlund, Sikström, Loftesnes y SonugaBarke, 2010, p. 56). Estudiantes con problemas de atención, incluyendo a aquellos con Trastorno de Déficit de Atención, podrían beneficiarse (Söderlund, Sikström, Loftesnes y Sonuga-Barke, 2010, p. 55). El modelo Moderate Brain Arousal (MBA) propone que cuando hay un nivel bajo de ruido cerebral, es necesario adicionar ruido externo para poder llegar, mediante el sistema perceptual, a un nivel óptimo de ruido interno (Sikstrom y Soderlund, 2007). En la última década investigaciones presentaron nuevas posibilidades generando inquietudes que conducen a la pregunta más importante: cómo el ruido blanco o aleatorio afecta el cerebro. Dentro del área educativa, investigaciones realizadas en los últimos diez años, indican que una cantidad de ruido blanco moderado puede beneficiar a alumnos y alumnas que poseen problemas de distracción e hiperactividad (Helps, Bamford, Sonuga-Barke y Söderlund, 2014; Instituto de Educación Secundaria Zapatón, 2011-12; Söderlund et al., 2010). Para poder explicar este mejoramiento de la atención, se hace una relación entre el ruido blanco y la presencia de niveles de dopamina, un neurotransmisor, en el cerebro (Söderlund et al., 2010). La dopamina es importante para la atención, la concentración y la memoria. En el cerebro hay muchos neurotransmisores. Para que el cerebro funcione de una manera normal, es necesario que haya cantidades suficientes de cada uno. La dopamina, por medio de las vías dopaminérgicas, ayuda a enviar la información recibida a distintas partes del cerebro. Las funciones ejecutivas (actividades y procedimientos mentales,

\footnotetext{
${ }^{2}$ Niveles óptimos de ruido blanco varía de una persona a otra.

${ }^{3}$ Un nivel suficiente de ruido podría ser necesario para que el sistema nervioso funcione de manera normal, a través del proceso de resonancia estocástica.

${ }^{4}$ Cuando el ruido auditivo ayuda a detectar una señal visual.
} 
determinar y llevar a cabo diferentes trabajos) que requieren tareas superiores, utilizan niveles más altos de este neurotransmisor. Se cree que el ruido blanco externo en el sistema nervioso no actúa de una manera similar a la dopamina, pero causa resultados similares al facilitar la transmisión de señales en el cerebro. Por lo tanto, se podría decir que para algunos estudiantes una cantidad de ruido sería necesario para que el sistema nervioso funcione de una manera óptima.

\section{Objetivo}

Objetivo General: Averiguar si el ruido blanco de fondo tiene un efecto positivo en la adquisición de una segunda lengua. Los objetivos específicos son: establecer la correlación de los resultados entre la presencia y la ausencia del ruido blanco de fondo entre el grupo experimental y el grupo de control, y observar y determinar si la presencia o ausencia de ruido blanco de fondo muestra alguna diferencia entre las tres habilidades: visual-auditiva, comprensión auditiva y comprensión lectora.

\section{Hipótesis}

Tres hipótesis nulas y tres alternativas fueron desarrolladas para contestar la pregunta de investigación.

$\mathrm{H}_{\mathrm{A}} 1$ : El uso de ruido blanco de fondo, facilita la comprensión auditiva.

$\mathrm{H}_{0} 1$ : El uso de ruido blanco de fondo, no facilita la comprensión auditiva.

$\mathrm{H}_{\mathrm{A}}$ 2: El uso de ruido blanco de fondo, facilita la comprensión visual-auditiva.

$\mathrm{H}_{0}$ 2: El uso de ruido blanco de fondo, no facilita la comprensión visual-auditiva.

$\mathrm{H}_{\mathrm{A}} 3$ : El uso de ruido blanco de fondo, facilita la comprensión lectora.

$\mathrm{H}_{0}$ 3: El uso de ruido blanco de fondo, no facilita la comprensión lectora.

\section{Variables}

Una variable independiente es aquella que hipotetiza causar una diferencia que se puede observar (Gall, Gall y Borg, 1999). En este estudio la variable independiente es la condición de ruido blanco de fondo (con ruido/ sin ruido). En cambio, la variable en la cual la diferencia es observada cuando la variable independiente es manipulada es la variable dependiente (Gall et al., 1999). En este estudio las variables dependientes son los resultados de las pruebas de comprensión visual-auditiva, visual y lectora. Manipular la variable independiente puede causar un efecto observable en las variables dependientes. La variable final en este diseño es la prueba de vocabulario dada al principio de la investigación. Esta variable será asignada como covariable usada para controlar estadísticamente diferencias entre alumnos y alumnas que pudieran estar presentes y pudieran confundir las diferencias entre los dos grupos.

\section{Método}

Este estudio causal-comparativo está diseñado para determinar si el uso del ruido blanco de fondo facilita la adquisición de una segunda lengua, lo cual es medido en la comprensión visual-auditiva, auditiva y lectora. La pregunta que esta investigación está contestando es: ¿Facilita el uso del ruido blanco de fondo la adquisición de una segunda lengua? 


\section{Población}

De un total de 250 estudiantes, se escogieron de manera aleatoria 60 estudiantes de escuela secundaria para este experimento. Estos números son adecuados para llevar a cabo la estadística inferencial, basado en el teorema de límite central (Virtual Laboratories in Probability and Statistics, 2015). Dos clases, 30 estudiantes cada una, de español nivel básico; una clase es el grupo de control y la otra clase es el grupo experimental. Ambas clases están expuestas a las mismas instrucciones, el mismo material, la misma cantidad de tiempo, las mismas evaluaciones. La clase del grupo experimental está expuesta a ruido blanco de fondo durante todo el experimento. La clase del grupo de control no está expuesta al ruido blanco de fondo en ningún momento.

A continuación se explican los pasos seguidos en esta investigación:

1. Se escogieron del curso de español nivel II $^{5} 200$ palabras. Estas palabras se utilizaron para crear los materiales para este experimento.

2. Durante tres meses, agosto, septiembre y octubre, se enseñaron las 184 palabras que forman parte de este experimento utilizando la metodología "Enseñar el dominio del lenguaje a través de la lectura y las historias contadas- Teaching Proficiency Through Reading and Storytelling” (TPRS).

3. Se escoge el grupo experimental al azar, utilizando una moneda.

4. Antes de comenzar la investigación:

- $\quad$ Cada estudiante escoge el nivel de ruido de fondo que más le favorece (Ver p. 5: Nivel de ruido blanco para cada alumno y alumna antes de comenzar la investigación).

- $\quad$ En los materiales creados para este experimento, se incluye el ruido de fondo que cada estudiante del grupo experimental ha escogido; creando materiales diferenciados según la necesidad de cada alumno y alumna.

- Antes de comenzar el experimento se da una prueba para evaluar las 200 palabras, de las cuales 184 ya se han enseñado. Se les informa a los estudiantes que solo deben demostrar lo que saben. También, que habrá palabras que no conocen porque todavía no se han enseñado. Este examen inicial es considerado en este diseño como variable final.

5. Se comienza el experimento que dura 4 días, de lunes a jueves.

6. Cada día al estudiante se le enseña 4 palabras de vocabulario por medio de un video (Ver p. 6: Descripción de la lección de video).

7. Se le da a cada estudiante tres evaluaciones por día: comprensión visualauditiva, comprensión auditiva y comprensión lectora. Todos los alumnos y alumnas reciben las mismas evaluaciones. La clase experimental se evalúa, con ruido blanco de fondo; la clase de control, sin ruido blanco de fondo.

8. Después de finalizar el experimento, se les da a los estudiantes un cuestionario para auto evaluar su experiencia.

\footnotetext{
${ }^{5}$ Nivel II, pertenece al nivel "Novice", básico o principiante, dentro de la escala de competencia lingüística (WWW.ACTFL.ORG)
} 
Nivel de ruido blanco para cada alumno y alumna antes de comenzar la investigación: se da a cada estudiante una evaluación de ruido blanco de fondo en donde el estudiante escoge el nivel que él mismo considere más confortable. Para poder lograrlo, se graba en audio una pequeña historia que la profesora lee y que contiene palabras de vocabulario que se han enseñado durante el semestre, en otras palabras, que ya los alumnos y alumnas conocen. Se utiliza un "pop filter", un accesorio de forma circular, que actúa como un filtro de protección que se coloca enfrente de un Micrófono de Estudio, mientras la profesora lee la historia. Este "pop filtro" se utiliza para todo tipo de grabación oral. La voz grabada es diseñada de tal forma que permite que la salida de la voz oscile entre los 70 y $80 \mathrm{~dB}^{6}$ a través de todo el estudio. Se utilizan siete niveles de sonido blanco de fondo, de $45 \mathrm{~dB}$ hasta $75 \mathrm{~dB}$. Los niveles de sonido blanco de fondo aumentan en incrementos de $5 \mathrm{~dB}$. Estos niveles se colocan sobre la voz de la profesora en un audio pre-grabado creando de esta forma siete audios que contienen el mismo nivel de voz sobre siete niveles diferentes de ruido blanco de fondo.

Lección del día en video ${ }^{7}$ : para poder enseñar las lecciones de cada día, cuatro días, se crean cuatro videos de audio. Se utilizan dos cámaras, Canon 5D MK II y Canon 5D MK III, Sony Wireless Lavalier Mic, micrófono que es conectado a Zoom H6N para poder grabar el audio. Se utilizan los programas Adobe Audition para el audio y Adobe Premier para grabar el video. El mismo video (uno por cada día) es usado para crear ocho nuevos videos. A sólo siete videos se les adicionan diferentes niveles de ruido blanco de fondo; dado que, el video usado para el grupo de control carece de ruido blanco de fondo. Importante destacar que se realiza el mismo proceso para cada uno de los videos; dado que, hay una lección diferente por cuatro días. Una vez que el audio o el video son grabados se utilizan filtros que posee el programa para limpiar el audio. Se usan tres diferentes filtros para garantizar un control de nivel absoluto. Primero se utiliza, Noise Reduction Filter, para eliminar todo tipo de ruido de fondo. Segundo, Speech Volume Leveler, para que todos los niveles vocales estén al mismo nivel. Tercero, Hard Limiter Filter, para que $80 \mathrm{~dB}$ sea el límite de las cuerdas vocales. Finalmente, se usa el Digital Sound Level Meter para medir el nivel de ruido blanco de fondo que sale del audífono que va a utilizar el estudiante en la clase. Esto asegura que el output o ruido blanco de fondo que se está escuchando esté al nivel establecido por cada estudiante antes de comenzar la investigación. Todos los estudiantes utilizan el mismo tipo de audífonos. Todo el material creado (28 videos con diferentes niveles de ruido de fondo y 4 videos sin ruido de fondo) se descarga en diferentes iPods; es decir: la lección en video, la historia de comprensión auditiva y el tiempo de duración para la lectura y comprensión ya pre-establecido. Cada iPod contiene las mismas lecciones; la única diferencia sería los diferentes niveles de ruido blanco de fondo diferenciados para cada estudiante dentro del grupo experimental y la ausencia de ruido blanco de fondo para el grupo de control.

Descripción de la lección de video: se lee la palabra, se parea la palabra con la ilustración y se traduce la palabra al inglés. La palabra de vocabulario que se enseña se coloca dentro de una o dos oraciones. Se traduce la oración completamente, también se usa para formular repeticiones "circling" (Ver Anexo 1) parte de la metodología TPRS. Se formulan preguntas en las que se utiliza la palabra dentro de la pregunta y de la

\footnotetext{
${ }^{6} \mathrm{~dB}$ decibeles: El decibel o decibelio es la unidad de medida de la intensidad sonora.

${ }^{7}$ Los niveles de ruido blanco de fondo, el audio de todas las grabaciones, y los videos, son desarrollados por Santitan Studios; corporación establecida en el año 2011. Esta firma se dedica a la producción de audio, videos, y medios de comunicación. www.santitanstudios.com.
} 
respuesta. Se repite cada una de las palabras nuevas de vocabulario la misma cantidad de veces dentro de la lección. La lección de video dura aproximadamente 18 minutos. El estudiante no puede escribir nada de lo que escucha. Esta información se le es dada al principio de la lección. El estudiante adquiere la palabra por medio de las repeticiones. Para asegurarse de que no hubiera ningún tipo de malentendido o comprensión parcial, se utilizan ilustraciones que muestran o que permiten asociar el significado de la palabra; también, se traduce la palabra, la frase, o la oración al inglés (Ray y Seely 2012, pp. 10,14). Esta asociación visual, auditiva-comprensiva y repetitiva hace que la palabra sea adquirida. Es importante destacar que el número de repeticiones varía según cada persona. Hay estudiantes que con tan solo pocas repeticiones ya han adquirido la palabra; mientras que otros, necesitan muchas más. Durante este período, el alumno y la alumna si lo desea, mientras escucha la lección en video, puede repetir en voz baja lo que dice el profesorado, contestar oralmente también en voz baja las preguntas que son formuladas o simplemente escuchar, leer y tratar de recordar cada palabra en el orden que se van presentando. Lo que se busca es que el alumno o alumna se sienta cómodo/a con la lección para evitar variables afectivas (Krashen, 2003, p. 6). Una vez que la lección de video concluye se dan las instrucciones que estudiante debe seguir. Las instrucciones son dadas en inglés solamente; esto evita posibles distracciones y malentendidos (ver anexo 3). El estudiante también tiene las mismas instrucciones escritas en inglés y español. Los siguientes pasos son las evaluaciones de comprensión visual-auditiva, comprensión auditiva y comprensión lectora.

- Instrumento para prueba visual-auditiva: después de ver y escuchar la lección del día en video, el alumno o alumna toma una prueba visual-auditiva. El estudiante escucha una de las cuatro palabras de vocabulario enseñadas y la parea con una de las cuatro ilustraciones que se muestran en el video. Cada ilustración contiene una letra del alfabeto. El alumno o alumna escribe la letra que identifica la palabra con la ilustración en una hoja de respuesta.

- Instrumento para prueba de comprensión auditiva: antes de escuchar una breve historia, aproximadamente 80 palabras, el estudiante tiene un minuto para leer cuatro declaraciones relacionadas con la historia que va a escuchar. Estas declaraciones están escritas en la hoja de respuesta, o instrumento de evaluación. Después de un minuto, el estudiante escucha dos veces un audio que contiene una breve historia. Al finalizar el audio, el estudiante escribe la letra " $\mathrm{A}$ " para indicar que la declaración es verdadera o la letra "B" para indicar que la declaración es falsa en el espacio establecido sobre la hoja de respuesta. Cada declaración contiene una de las palabras enseñadas.

- Instrumento para prueba de comprensión lectora: Cada día, el estudiante lee una historia diferente de aproximadamente 155 a 160 palabras. Esta historia es parte de la evaluación de comprensión lectora. Al final de la historia se adjuntan cuatro declaraciones. Cada declaración contiene una de las palabras de vocabulario enseñadas en la lección del día; también, cada declaración busca la relación afirmativa o negativa en correlación con la historia. El alumno y alumna marca en el área indicada sobre la hoja de respuesta, o instrumento de evaluación, la letra "A", si la respuesta es verdadera o la letra "B", si la respuesta es incorrecta. 


\section{Resultados}

\section{Descripción de la muestra}

La edad promedio de ambos grupos es de 14 años. La media en el grupo de control es de 14.7 y en el grupo experimental es de 13.8 (Ver Tabla 1). Hay más mujeres en el grupo experimental, totalizando un $64 \%$ de los estudiantes; mientras que, hay más hombres en el grupo de control, con un total del 59\% de todos los estudiantes.

Tabla 1

Descripción de la Muestra según la condición de Fondo

\begin{tabular}{|c|c|c|c|c|}
\hline & \multicolumn{2}{|c|}{ Sin Sonido $(n=27)$} & \multicolumn{2}{|c|}{ Con Sonido $(n=25)$} \\
\hline & $n$ & $\%$ & $n$ & $\%$ \\
\hline \multicolumn{5}{|l|}{ Características } \\
\hline \multicolumn{5}{|l|}{ Edad } \\
\hline 13 & 0 & 0.0 & 1 & 4.0 \\
\hline 14 & 17 & 63.0 & 17 & 68.0 \\
\hline 15 & 5 & 18.5 & 3 & 12.0 \\
\hline 16 & 1 & 3.7 & 3 & 12.0 \\
\hline Mayor de 16 & 4 & 14.8 & 1 & 4.0 \\
\hline \multicolumn{5}{|l|}{ Género } \\
\hline Female & 11 & 40.7 & 16 & 64.0 \\
\hline Male & 16 & 59.3 & 9 & 36.0 \\
\hline
\end{tabular}

Nota: Fuente: D’Astoli Santaiti (2015)

Los datos fueron introducidos en el paquete estadístico para ciencias sociales (SPSS). Los datos fueron analizados asumiendo valores normales y valores atípicos. Un análisis multivariado de los datos encontró un valor atípico multivariante. A sí mismo, una examinación de las variables por grupo encontró siete valores atípicos adicionales. Después de haber removido estos valores atípicos, se utilizaron 52 casos para analizar la pregunta de esta investigación. Las medias y desviaciones estándar de las variables dependientes y la covariable se presentan en la tabla 2. Estos valores se utilizaron para analizar la pregunta de investigación.

Tabla 2

La Media y la Deviación Estándar de las Variables Dependientes y la Covariable

\begin{tabular}{|c|c|c|c|c|c|c|c|c|}
\hline \multirow[b]{2}{*}{ Variable } & \multicolumn{4}{|c|}{$\begin{array}{c}\text { Sin Sonido } \\
\quad(n=27)\end{array}$} & \multicolumn{4}{|c|}{$\begin{array}{c}\text { Con Sonido } \\
\quad(n=25)\end{array}$} \\
\hline & Min & Max & $\mathrm{M}$ & SD & Min & Max & $\mathrm{M}$ & SD \\
\hline $\begin{array}{l}\text { Examen de } \\
\text { vocabulario } \\
\text { Comprensión }\end{array}$ & .44 & .92 & .72 & .15 & .54 & .96 & .75 & .12 \\
\hline Audio-visual & 14 & 16 & 15.52 & 0.84 & 14 & 16 & 15.30 & 0.83 \\
\hline Auditiva & 4 & 14 & 9.93 & 2.87 & 6 & 15 & 11.52 & 2.65 \\
\hline Lectora & 7 & 16 & 11.2 & 2.98 & 9 & 15 & 12.32 & 1.80 \\
\hline
\end{tabular}

Nota: Fuente: D’Astoli Santaiti (2015)

Las diferencias entre los dos grupos se determinaron mediante el uso de un único factor de análisis multivariado de covarianza (MANCOVA). La variable independiente 
fue los dos niveles de condición de fondo (con ruido/ sin ruido). Las variables dependientes fueron la medición de los resultados de comprensión audio-visual, comprensión auditiva y comprensión lectora. La covariable fue el examen de vocabulario dado al principio de la investigación. El nivel de significación fue $\alpha<.05$. La homogeneidad se midió mediante la prueba de Box de igualdad de matrices de covarianzas y fue hallada no significativa $(F=1.72, p=.112)$. La prueba de Levene de igualdad de varianzas de error no encontró problemas con los datos. La media marginal estimada para las variables dependientes se encuentra en la tabla 3.

Tabla 3

Media Marginal de las Variables Dependientes

\begin{tabular}{|c|c|c|c|c|c|c|c|c|}
\hline \multirow{3}{*}{$\begin{array}{l}\text { Variable } \\
\text { Dependiente }\end{array}$} & \multicolumn{4}{|c|}{$\begin{array}{c}\text { Sin Sonido } \\
(n=27)\end{array}$} & \multicolumn{4}{|c|}{$\begin{array}{l}\text { Con Sonido } \\
(n=25)\end{array}$} \\
\hline & \multirow[b]{2}{*}{$\mathrm{M}$} & \multirow[b]{2}{*}{ SE } & \multicolumn{2}{|c|}{$95 \% \mathrm{CI}$} & \multirow[b]{2}{*}{ M } & \multirow[b]{2}{*}{ SE } & \multicolumn{2}{|c|}{$95 \% \mathrm{CI}$} \\
\hline & & & Inferior & Superior & & & Inferior & Superior \\
\hline Audio-visual & 15.55 & .15 & 15.25 & 15.85 & 15.27 & .15 & 14.96 & 15.58 \\
\hline Auditiva & 10.05 & .46 & 9.13 & 10.97 & 11.39 & .48 & 10.43 & 12.34 \\
\hline Lectora & 11.33 & .42 & 10.48 & 12.17 & 12.21 & .44 & 11.33 & 13.09 \\
\hline
\end{tabular}

Nota: Fuente: D’Astoli Santaiti (2015)

Por medio del examen estadístico de Wilks Lambda se pudo determinar que el análisis multivariado fue significativo $(\mathrm{F}=2.92, \mathrm{p}=.044)$. Indicando que una o más de las medidas eran diferentes entre los grupos (ver tabla 4). A sí mismo, el análisis univariado encontró una medida, la comprensión auditiva, significativa $(\mathrm{F}=4.08, \mathrm{p}=$ .049). El grupo experimental (con ruido blanco de fondo) tuvo una puntuación significativamente más elevada $(\mathrm{M}=11.30)$ en comprensión auditiva que la que tuvo el grupo de control $(M=10.05)$. No se encontraron otras diferencias importantes entre los dos grupos. Demostrando de esta forma que, las hipótesis nulas 2 y 3 no fueron rechazadas, mientras que la hipótesis nula 1 fue rechazada.

Tabla 4

Resultados del Análisis de las Hipótesis de Investigación

\begin{tabular}{lcc}
\hline Type of test & $F$ & $p$ \\
\hline Multivariate & & \\
$\quad$ Wilks' lambda & 2.92 & .044 \\
Univariate & & \\
$\quad$ Audiovisual comprehension & 1.72 & .196 \\
Listening comprehension & 4.08 & .049 \\
$\quad$ Reading comprehension & 2.10 & .153 \\
\hline
\end{tabular}

Nota: Fuente: D’Astoli Santaiti (2015)

Después de completarse el estudio, los estudiantes respondieron a cuatro preguntas relacionadas al ruido blanco de fondo. Las respuestas a las preguntas fueron categorizadas utilizando la escala Likert de 5 puntos que van de 1 (nunca), 2 (algunas veces), 3 (a veces), 4 (la mayoría de las veces), 5 (siempre). Las respuestas de los estudiantes fueron promediadas a través de la escala Likert variando de 1 a 5 , con la puntuación más alta indicando que el ruido de fondo les ayudó a concentrarse, estudiar y aprender, y que preferían una clase con ruido de fondo. La tabla 5 contiene las medias y desviaciones estándar para cada grupo. En cada caso, el grupo experimental muestra 
puntuaciones más altas. Las medias del grupo experimental fueron estadísticamente superiores a las medias del grupo de control para cada ítem en el cuestionario.

Tabla 5

Resultado de la Encuesta en cuanto a Condición de Fondo

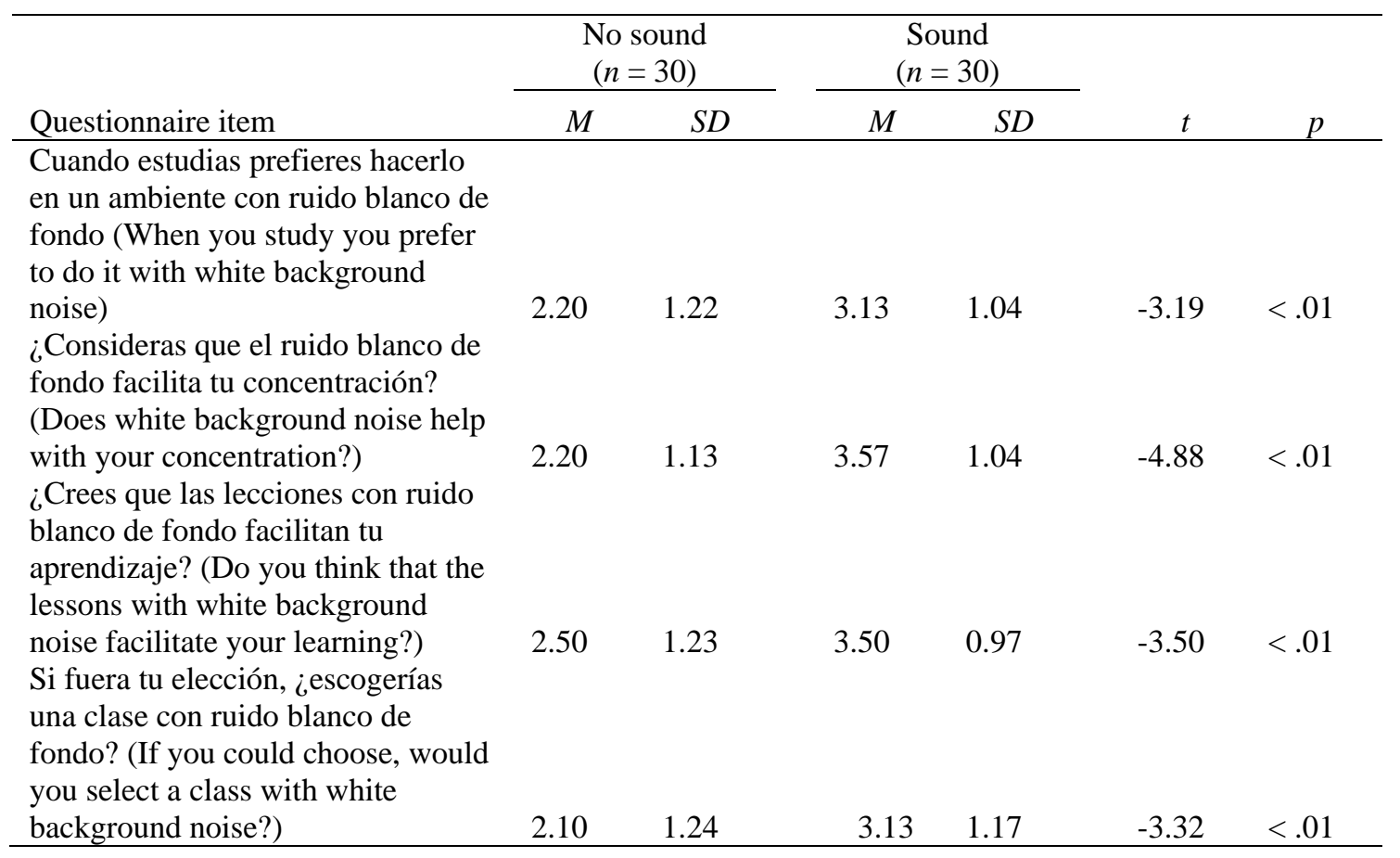

Nota: Fuente: D’Astoli Santaiti (2015)

\section{Discusión y conclusiones}

Dos clases de treinta alumnos cada una, 60 en total, una constituye la clase de control (sin sonido de fondo) y la otra, representa la clase experimental (con sonido de fondo). Ambas, fueron evaluadas en reconocimiento de palabras a través de la traducción y habilidades de comprensión para determinar si el uso del ruido blanco de fondo facilita la adquisición de una segunda lengua. La revisión de los datos encontró ocho valores atípicos, como resultado 52 casos fueron usados para analizar la pregunta de investigación. MANCOVA encontró que el grupo experimental (con sonido de fondo) tuvo una puntuación significativamente más alta que el grupo de control (sin sonido de fondo) en el área de comprensión auditiva. En este estudio se utilizó vocabulario de niveles básicos eliminando variables sociolingüísticas. El lenguaje es simple y está representado con ilustraciones y traducciones para evitar malentendidos. Las lecciones se desarrollaron en un ambiente confortable, Affective Filter Hypothesis (Krashen, 1992). Se le aclara al estudiante que solo debe demostrar lo que ha aprendido. Comparando los dos grupos, uno experimental y otro de control, los resultados demuestran que el ruido de fondo favoreció al grupo experimental dentro del área de comprensión auditiva. Analizando los resultados de las tres habilidades: comprensión audio-visual, comprensión auditiva y comprensión lectora en ambos grupos, grupo experimental y grupo de control, se establece que el uso del ruido blanco de fondo tanto en la habilidad de comprensión visual-auditiva, como en la habilidad de comprensión 
lectora, no ha demostrado facilitar ni perjudicar la adquisición de una segunda lengua; señalando que la diferencia entre ambos es insignificante. Las hipótesis nulas 2 y 3 no se rechazan; las cuales indican que el uso del ruido blanco de fondo en las áreas de compresión visual-auditiva y comprensión lectora no facilita la adquisición de una segunda lengua. Mientras que el uso del ruido blanco de fondo demostró favorecer ampliamente la comprensión auditiva; indicando una diferencia considerable a favor del grupo experimental. Por lo tanto, se rechaza la hipótesis nula 1, el ruido blanco de fondo no facilita la comprensión auditiva de una segunda lengua, y se acepta la hipótesis alternativa.

Aún no se conoce cómo el ruido aleatorio beneficia el cerebro. Existen diferentes teorías, pero ninguna todavía puede demostrarlo. El fenómeno de resonancia estocástica fue observado y estudiado en un gran número de investigaciones físicas y biológicas; pero, desde el punto de vista neuronal no existe hasta hoy una explicación concreta. Ningún estudio previo ha planteado, encarado y profundizado el efecto del ruido blanco en el aprendizaje de una segunda lengua. Esto ha limitado considerablemente los antecedentes y referencias incluidos en esta investigación. Resultados de estudios anteriores han demostrado que el ruido blanco a niveles altos, ayuda al estudiante hiperactivo, afectando negativamente al estudiante que no presenta problemas de aprendizaje. En el análisis de la curva U invertida utilizando el fenómeno de resonancia estocástica, (Sikstrom y Söderlund 2007, p. 841) comprueban que "performance peaks at a moderate noise level" ; por lo tanto, deduce que, mucho o poco ruido, afecta el rendimiento. Este modelo también sugiere que el punto máximo de la curva de resonancia estocástica depende del nivel de dopamina. En el año 2010, se propuso un nuevo modelo (Söderlund et al., 2010) utilizando solamente dos niveles de ruido blanco. Fue la primera vez que se estudió cómo el ruido blanco afecta el rendimiento cognitivo de niños con diferentes habilidades de atención. Este modelo indicó que el fenómeno de resonancia estocástica varía de una persona a otra. También, reveló que la dopamina juega un papel muy importante; variando el nivel de este neurotransmisor, de una persona a otra. Los resultados demostraron que la dopamina influye con el cumplimiento de las diferentes tareas dependiendo de la dificultad de las mismas. Cuanto más atención y memoria de trabajo se necesite, más dopamina requiere el cerebro (Söderlund et al., 2010). En este estudio, la falta de atención fue el factor clave, no la hiperactividad.

Mediante muchos años de observación y de instrucción académica, uno puede afirmar que la comprensión auditiva es la habilidad más difícil en la enseñanza de una segunda lengua. "Estudiantes manifiestan que las dificultades se encuentran fundamentalmente en la habilidad de comprensión auditiva" (Cisterna-Zenteno, SotoHernandez y Díaz-Larenas 2016, p. 12). Esta habilidad requiere por parte del alumno y alumna una concentración y atención superior que las otras dos habilidades, la visual y la lectora. Siguiendo esta línea de pensamiento, al haber mayor necesidad de atención y concentración hay más necesidad de dopamina en el cerebro. Esto puede explicar la diferencia en los resultados obtenidos.

Uso práctico dentro de la clase: Es importante para el aprendizaje de una segunda lengua que el estudiante utilice todas las habilidades que forman parte del Input, es decir lo que vemos y oímos; por lo tanto, es necesario trabajar con la comprensión visual, la comprensión auditiva y la comprensión lectora. Dado que se ha demostrado que el ruido de fondo ayuda a la comprensión auditiva, una sugerencia

\footnotetext{
${ }^{8} \mathrm{El}$ desempeño se maximiza a un nivel de ruido moderado.
} 
práctica y posible dentro del aula sería usar la tecnología para incluir el ruido blanco de fondo en las prácticas o lecciones de comprensión auditiva. Por ejemplo, utilizando el programa Audacity, el enseñante puede grabar historias y adicionar diferentes niveles de ruido blanco de fondo dejando que cada estudiante escoja el nivel que más le favorezca. El enseñante solamente necesitaría un medidor de sonido "Digital Sound Level Meter" (un aparato que mide la salida del ruido, es decir lo que estaría escuchando el alumno y alumna en decibeles) y el programa Audacity, u otro programa, que permitiera adicionar ruido de fondo dentro del material que haya sido grabado. Otra posible diferenciación que el educador pudiera considerar, sería el uso del ruido de fondo en el aula; por ejemplo, el ruido de la lluvia dentro de la clase como algo opcional. Considerando que los resultados indicaron que no beneficia ni perjudica la comprensión visual-auditiva y comprensión lectora, se dejaría a criterio de cada clase y de cada situación. Muchas veces existen, dentro del aula, situaciones donde el enseñante no tiene una forma simple y directa para resolverlas; generalmente, el reto más grande para el docente es tener dentro de una clase estudiantes hiperactivos y distraídos. Esta experiencia podría ayudar al estudiante a conocerse mejor a sí mismo y entender que existen diferentes maneras de aprender el material; como también, evitar la distracción y de esta manera poder concentrarse. El profesorado se iría convirtiendo en una persona que facilita el proceso de enseñanza ayudando al estudiante a ser consciente de sus propias estrategias de aprendizaje. Además, el uso de ruido blanco en un estudio piloto, (Söderlund, Björk y Gustafsson, 2016) indicó que niños y niñas con trastorno de déficit de atención con hiperactividad (ADHD) no medicados rindieron más que aquellos medicados en cuanto al desempeño de tareas. En nuestras aulas podemos ver que a muchos de nuestros estudiantes los tratamientos farmacológicos no les favorecen. A veces, se tarda todo un semestre hasta encontrar la dosis justa que pueda beneficiarlos. También, tenemos un grupo de padres y madres que no creen en el uso de medicamentos para mejorar la atención. Es necesario destacar que el estudiante tiene que ser parte de la decisión en cuanto al uso del ruido blanco de fondo como posible instrumento de ayuda; pudiéndose utilizar como una posible medida de diferenciación. A veces, no hay un síntoma que lleve a pensar que esto sea necesario para ciertos estudiantes. Este estudio demostró que hubo estudiantes que escogieron un nivel de ruido blanco de fondo a $65 \mathrm{~dB}$. Estos jóvenes no demostraron ningún tipo de hiperactividad; todo lo contrario. Sin embargo, indicaron después del experimento que el ruido les permite estar más concentrados.

Futuras investigaciones podrían desarrollarse exponiendo al mismo estudiante a lecciones con ruido blanco de fondo o sin ruido blanco de fondo; de esta forma, ver cómo el ruido beneficiaría a cada persona de manera individual. Sería importante investigar enseñando con ruido o sin ruido de fondo en otro idioma que no sea el español. Desde el punto de vista neurológico, el/la docente, necesita saber cuánto tiempo el estudiante puede estar expuesto al ruido blanco de fondo durante el día escolar, o durante las horas de estudio fuera de la escuela, mientras el estudiante hace su tarea, y necesita concentrarse. Es necesario destacar que solo se ha analizado el Input. Investigaciones futuras deberían medir también el Output, es decir, la participación oral y la escrita. También, es importante subrayar que recién se están comenzando a observar los posibles beneficios y que queda mucho por averiguar para poder llegar a entender el efecto del ruido blanco en el cerebro; por lo tanto, es fundamental seguir investigando. 


\section{Referencias}

Aledo, J.C. (2007). Ruido Vital. Encuentros en Biología. Universidad de Málaga, 120, (2007), 5-7.

Álvarez Miño, L., y Fajardo Fajardo, D. (2002). Diseño y Desarrollo de una práctica de laboratorio. Conceptos Básicos de Resonancia Estocástica. Revista Colombiana de Física, 34 (1), 305- 306.

Baijot, S., Slama, H., Söderlund, G., Dan, B., Deltenre, P., Colin, C. \& Deconinck, N. (2016). Neuropsychological and neurophysiological benefits from white noise in children with and without ADHD. Behavioral and Brain Functions : BBF, 12, 11. doi: 10.1186/s12993-016-0095-y

Cisterna-Zenteno, C., Soto-Hernández, V., y Díaz-Larenas, C. (2016). Medición de habilidades de comprensión lectora y auditiva en estudiantes de Pedagogía en Inglés de una universidad chilena. Revista Electrónica Educare, 20 (1), 1-21. doi: $10.15359 /$ ree. $20-1.8$

Dolores, M. (10 de noviembre, 2015) Ruido luminoso en el cerebro. UNAM, Recuperado de: http://www.luz2015.unam.mx/leer/223/ruido-luminoso-en-elcerebro

Faisal, A, Selen, L. \& Wolpert, D. (2008). Noise in the nervous system. Nature Reviews Neuroscience, 9, 292-303. doi: 10.1038/nrn2258

Gall, J.P., Gall, M.D, \& Borg, W.R. (1999). Applying Educational research: A practical guide (4th ed.). New York, NY: Addison Wesley Longman.

Hong, D., Martin, J. \& Saidel, W. (2006). The mechanism for stochastic resonance enhancement of mammalian auditory information processing. Theoretical Biology and Medical Modeling, 3, 39. doi: 10.1186/1742-4682-3-39

Instituto de Educación Secundaria Zapatón. Consejería de educación, Cultura y Deporte. Gobierno de Cantabria (Curso 2011-2012) Proyecto de innovación tecnológica en formación profesional. Beneficios del ruido. Recuperado de https://www.ieszapaton.es/ieszapaton/NUEVA_WEB/PROYECTOS/b_ruido.pd $\mathrm{f}$

Krashen, S. (2003). Exploration in Language Acquisition and Use. En S. Krashen, Principles of Language Acquisition. (p. 6). Portsmouth, NH: Heinemann.

Lugo, E., Doti, R. \& Faubert, J. (2008). Ubiquitous Cross modal Stochastic Resonance in Humans: Auditory Noise Facilitates Tactile, Visual and Proprioceptive Sensations. Plos-One, 3 (8), e2860. doi: 10.1371/journal.pone.0002860

McDonnell MD \& Abbott D (2009) What Is Stochastic Resonance? Definitions, Misconceptions, Debates, and Its Relevance to Biology. PLoS Comput Biol 5(5), e1000348. doi: 10.1371/journal.pcbi.1000348

Mendez-Balbuena, I., Manjarrez, E., Schulte-Monting, J., Huethe, F., Tapia, J., HeppReymond, MC. \& Kristeva,R. (2012). Improved Sensorimotor Performance via Stochastic Resonance. The Journal of Neuroscience: the Official Journal of the Society for Neuroscience, 32(36), 12612-12618. doi: 10.1523/JNEUROSCI.0680-12.2012

Ray, B. \& Seely, C. (2012). Fluency through TPR Storytelling. Achieving real language acquisition in school. En B. Ray y C. Seely, TPRS Essential Concepts and 
Practices (pp.14, 15). Eagle Mountain, UT: Command Performance Language Institute y Blaine Ray Workshops.

Russell,D., Wilkens, L. \& Moss, F. (1999). Use of behavioral stochastic resonance by paddle fish for feeding. Nature, 402, 291-294. doi: 10.1038/46279

Seo, NJ., Kosmopoulos, MI., Enders, LR \& Hur, P. (2014). Effect of Remote sensory Noise on Hand Function Post Stroke. Frontiers in Human Neuroscience, 8 (934), 1-9. doi: 10.3389/fnhum.2014.00934

Söderlund , G., Bjork, C. \& Gustafsson, P.(2016). Comparing Auditory Noise Treatment with Stimulant Medication on Cognitive Task Performance in Children with Attention deficit Hyperactivity Disorder: Results from Pilot Study. Front. Psychology, 7, 1331. doi: 10.3389/fpsyg.2016.01331

Söderlund et al. (2010). The effects of background White noise on memory performance in inattentive school children. Behavioral and Brain Functions: $B B F, 6$, 55. doi: 10.1186/1744-9081-6-55

Söderlund, G., Sikström, S. \& Smart, A. (2007). Listen to the noise: noise is beneficial for cognitive performance in ADHD. Journal of Child Psychology and Psychiatry, 48, (8), 840-847. doi: 10.1111/j.1469-7610.2007.01749.x

Toral, R. (2002). No hay ruido bueno, pero algunos son mejores que otros. Revista Española de Física 16 (3), 60-62.

Virtual Laboratories in Probability and Statistics (2015). The central limit theorem. Huntsville, AL: Department of Mathematical Sciences, University of Alabama at Huntsville. Recuperado de http://www.math.uah.edu/stat/sample/CLT.html

Fecha de recepción: 10/07/2017

Fecha de revisión: 30/08/2017

Fecha de aceptación: 16/10/2017 